Itinéraires Itinéraires

Littérature, textes, cultures

\title{
Lectures communistes de Stendhal : enjeux politiques et patrimoniaux
}

\section{François Vanoosthuyse}

\section{(2) OpenEdition}

\section{Journals}

Édition électronique

URL : http://journals.openedition.org/itineraires/1411

DOI : 10.4000/itineraires. 1411

ISSN : 2427-920X

Éditeur

Pléiade

\section{Édition imprimée}

Date de publication : 1 décembre 2011

Pagination : 117-133

ISBN : 978-2-296-55744-4

ISSN : 2100-1340

\section{Référence électronique}

François Vanoosthuyse, "Lectures communistes de Stendhal : enjeux politiques et patrimoniaux», Itinéraires [En ligne], 2011-4 | 2011, mis en ligne le 01 décembre 2011, consulté le 19 avril 2019. URL http://journals.openedition.org/itineraires/1411; DOI : 10.4000/itineraires.1411

\section{(C) $(\oplus \Theta$}

Itinéraires est mis à disposition selon les termes de la licence Creative Commons Attribution - Pas d'Utilisation Commerciale - Pas de Modification 4.0 International. 


\title{
Lectures communistes de Stendhal : enjeux politiques et patrimoniaux
}

\begin{abstract}
This article seeks to evaluate Stendhal's reception by French communist intellectuals, particularly Aragon, Andrieu, and Berberis. This critical reading is contextualized both historically and intellectually, in order to contribute to the study of Stendhal's social and political ideas, as well as the cultural history of the French Communist Party.
\end{abstract}

Keywords : Stendhal, Stendhalian critique, history of the French Communist Party, cultural heritage, socio-political reception

Mots clés : Stendhal, critique stendhalienne, histoire du Parti communiste français, patrimoine culturel, sociopolitique de la réception

En 1983, à l'occasion du bicentenaire de la naissance de Stendhal, les éditions Jean-Claude Lattès firent paraître Stendhal ou le Bal masqué de René Andrieu, membre du Comité central du PCF, rédacteur en chef de L'Humanité ${ }^{1}$. La même année, Pierre Barbéris ${ }^{2}$ publia Sur Stendhal aux Éditions sociales, ouvrage qui regroupait ses présentations des œuvres de l'écrivain au Livre Club Diderot en 1975, ainsi que sa communication au colloque du bicentenaire à Grenoble, "Stendhal et l'avenir ». D'un nouveau complot contre les industriels, pamphlet de Stendhal datant de 1825 , était intégralement reproduit dans ce volume. Peu de temps avant l'édition du Livre-Club Diderot, Stendhal avait fait pour la première fois

1. La quatrième de couverture présente ainsi l'auteur : « né en 1920 dans un petit village du Lot. Mobilisé en 1940. Après l'armistice, maître d'internat à Toulouse. Entre dans la Résistance. Prépare l'agrégation. Écrit un diplôme... sur Stendhal. Adhère au Parti communiste en 1942. Maquisard dans le Lot en 1943. Journaliste à la Libération. À Ce Soir, puis à L'Humanité, dont il devient rédacteur-en-chef en 1958. Membre du Comité central du Parti communiste ». 2. Professeur de littérature française, grand et décapant balzacien, président-fondateur de la Société des études romantiques (1969-1971). Pierre Barbéris militait au Parti communiste à l'époque de ces publications. 
l'objet d'un numéro de la revue Europe, alors d'obédience communiste ${ }^{3}$ (numéro 521, juillet-septembre 1972).

Ces textes des années 1970 et 1980 ont un ancêtre commun, La Lumière de Stendhal, publié par Aragon en 1954 aux éditions Denoël. Ce livre réunit différentes études et articles écrits depuis 1948 au sujet de Stendhal, mais aussi de Zola, de Desbordes-Valmore ou Barrès. Il fait partie d'une importante série de textes qu'Aragon consacra dans les années 1950 à des écrivains et à des artistes du patrimoine français, parmi lesquels on retiendra en particulier L'Exemple de Courbet (Cercle d'art, 1952) et Hugo poète réaliste (Éditions sociales, 1952).

L'objet de cet article est de rendre compte, en nous fondant principalement sur l'étude des ouvrages d'Aragon, Andrieu et Barbéris, des rapports que des intellectuels communistes français ont entretenus avec l'œuvre de Stendhal dans la seconde moitié du $\mathrm{xx}^{\mathrm{e}}$ siècle - plus précisément sur la période 1954-1983.

Notre corpus n'est pas exhaustif : il faudrait notamment consulter ce qui concerne Stendhal dans la revue La Nouvelle Critique. Revue du marxisme militant (publiée aux Éditions sociales entre 1948 et 1980). Mais il faudrait aussi se demander a contrario pourquoi Stendhal ne fait pas partie, par exemple, des corpus littéraires pourtant largement dix-neuviémistes analysés par des intellectuels d'obédience marxiste comme Jacques Rancière ou Pierre Macherey. Notre corpus n'est pas non plus homogène. Non seulement parce que chaque auteur (un écrivain, un journaliste, un professeur) occupe dans le champ de la littérature et de ses dérivés une position différente, mais aussi parce que leurs analyses et leurs options politiques se distinguent. C'est une histoire du stendhalisme mais aussi une histoire culturelle et littéraire du PCF qui s'esquissent à l'analyse de ce corpus.

Au moment où cette histoire commence, il existe déjà une tradition stendhalienne de gauche, dont le titre le plus célèbre est celui de Léon Blum, Stendhal et le beylisme (juillet 1914; rééditions chez Albin Michel en 1930 et 1947; en 2000 pour la dernière). Avant le texte de Blum avait paru la célèbre version zolienne de Stendhal ${ }^{4}$, où les louanges, à caractère politique (tournées

3. Europe est dirigée à l'époque par Pierre Abraham. Pierre Gamarra (qui la dirige à partir de 1974), Louis Aragon et Elsa Triolet font également partie du comité de rédaction. Notons que Stendhal apparaît tardivement dans le catalogue d'Europe : pour nous en tenir aux écrivains du XIX ${ }^{\mathrm{e}}$ siècle, Balzac, Zola, Sand, Lamennais, Gogol, Heine, Rimbaud, Nerval, Vallès, Flaubert, Courteline avaient été abordés au cours des années 1950; Tolstoï, Les Misérables, Delacroix, Balzac, Courier, Baudelaire, Constant, Zola, Maupassant, Lamartine, Flaubert, Dickens au cours des années 1960; Dumas père, Dostoïevski, Nerval entre 1970 et le printemps 1972.

4. Voir Émile Zola, Du roman. Sur Stendhal, Flaubert et les Goncourt, préface de Henri Mitterand, Bruxelles, Éditions Complexe, 1989. 
contre Sainte-Beuve et contre la critique conservatrice), étaient balancées par une appréciation négative, selon laquelle Stendhal n'avait pas suffisamment rendu compte des milieux, avait été insuffisamment matérialiste. Cette critique de fond, qui était une critique de gauche, fut passionnément discutée par les stendhaliens, y compris par des stendhaliens d'obédience marxiste, ou par des marxistes intéressés à Stendhal, comme Lukacs ${ }^{5}$. Aragon pour sa part constitue dans son livre un attelage original, celui de Stendhal et de Zola, le second constituant un complément nécessaire au premier. La persistance d'un stendhalisme de gauche, et même la grande influence de Stendhal sur des écrivains engagés à gauche entre les deux guerres et au-delà, ne fait aucun doute. Citons notamment les noms d'Alain ${ }^{6}$, Malraux, Sartre, Prévost, Camus ${ }^{7}$. Mais, au moment où l'ouvrage d'Aragon parait, c'est sans doute le stendhalisme de droite, et même d'extrême droite, qui est le plus en vue. On sait que Stendhal est une grande référence de Drieu par exemple. L'ouvrage de Maurice Bardèche, Stendhal romancier, a paru en 1947 à La Table ronde (réédition en 1969 : autre moment opportun pour opposer Stendhal au socialisme). La Revue critique des idées et des livres d'Eugène Marsan, proche de l'Action française, avait fait régulièrement du temps de la jeunesse d'Aragon la promotion de Stendhal et du stendhalisme. Y collaboraient Barrès, Maurras lui-même, Bourget, Léon Daudet, Jacques Bainville, Henri Martineau'. C'est la raison pour laquelle Aragon s'attaque d'entrée de jeu à la figure de Barrès (auteur de la définition de Stendhal comme "professeur d'énergie »), et construit face à celui-ci la figure d'un Stendhal prémarxiste.

Une autre tendance forte du stendhalisme des années 1940 et 1950 est incarnée par Henri Martineau (1882-1958) ${ }^{9}$, type même du critique érudit rempli d'une sympathique piété. Il publia par exemple au Divan en 1950 Le Calendrier de Stendhal, qui proposait, sous une forme proche de l'agenda, l'emploi du temps d'Henri Beyle, mois après mois, jour après jour, de son adolescence à sa mort, ainsi que les plans des lieux et des quartiers qu'il avait habités. Cet ouvrage, bien qu'exceptionnel dans sa

5. Georg Lukacs, Balzac et le réalisme français [1952], préface de Gérard Gengembre, Paris, La Découverte, 1999.

6. Alain, cofondateur du Comité de vigilance des intellectuels antifascistes en 1934, publie son Stendhal l'année suivante.

7. Pour une histoire complète de la critique stendhalienne, nous renvoyons à deux ouvrages : Stendhal, Mémoire de la critique, collectif, préface de Michel Crouzet, Paris, Presses universitaires Paris Sorbonne, 1995; Philippe Berthier, Stendhal en miroir. Histoire du stendhalisme en France (1842-2004), Paris, Champion, 2007.

8. René Andrieu publie en fin de volume des appréciations d'écrivains et de critiques de renom au sujet de Stendhal, dont deux extraits de Maurras et de Barrès datant respectivement de 1888 et de 1894 .

9. Son édition des romans parut à la Pléiade en deux volumes en 1947 et 1948 (l'année où sortit également La Chartreuse de Parme de Christian-Jaque, avec Gérard Philipe, et le premier texte d'Aragon sur Stendhal; c'est aussi l'année où La Nouvelle Critique. Revue du marxisme militant commença de paraître aux Éditions sociales). 
conception, est assez typique du stendhalisme votif. Martineau ne fait pas de Stendhal un usage politique : s'il est militant, c'est de Stendhal lui-même, de sa « philosophie », comme disait Rémy de Gourmont, de sa «psychologie », comme disait Paul Bourget, et de ses héros.

Il est intéressant de noter qu'un bon nombre d'articles parus dans la revue Europe en 1972 ont été conçus dans ce même esprit votif. Jacqueline Bruller-Dauxois y signe par exemple un « Pour Julien ». Annie-Claire Jaccard un « Julien Sorel : la mort et le temps du bonheur ». Les contributions de Franz Hellens et de Charles-Henry Reymont s'intitulent respectivement « Stendhal sinon le meilleur, le plus vrai » et « La sincérité de Stendhal ». À côté de ces articles, d'autres contributions, plus caractéristiques de l'esprit universitaire, constituent des synthèses sérieuses et politiquement peu marquées sur des sujets importants et classiques tels que Stendhal et l'Amérique, Stendhal et les sciences ou Stendhal et l'actualité. Seules les contributions d'ailleurs remarquables de l'historien Jacques Madaule et de Geneviève Mouillaud, universitaire, spécialiste de Stendhal, cherchent à penser Stendhal dans son contexte et à en proposer des lectures politiques. Il existe donc une certaine porosité entre tous les stendhalismes, y compris dans la sphère d'influence marxiste ou marxisante.

Mais les lectures d'Aragon, Andrieu et Barbéris se démarquent entre toutes par leur caractère intrinsèquement politique et ouvertement marxiste. Elles tranchent de ce point de vue sur le stendhalisme « intimiste », de gauche comme de droite. Cependant, il est manifeste que les auteurs ont une connaissance intime, précise et aussi sentimentale de Stendhal; ce qui leur permet de jouer sérieusement le jeu du débat littéraire et de la confrontation scientifique. Aragon et Andrieu, notamment, proposent des études informées, qui font écho aux travaux d'érudition de Liprandi ${ }^{10}$ et de Martineau, et dans une moindre mesure aux essais de Léon Blum et de Jean Prévost ${ }^{11}$. Aragon se montre même particulièrement savant, qui apporte des précisions sur un certain nombre de détails, ou d'aspects généralement traités comme des points de détail par la critique stendhalienne (son travail sur le contexte d'écriture et de publication du Rouge par exemple est remarquable, de même, dans une optique moins politique, que le parcours qu'il propose à travers les différentes versions italiennes, anglaises et françaises de 1 'histoire de Vittoria Accoramboni ${ }^{12}$ ).

10. Claude Liprandi fit paraître à Avignon en 1949 un ouvrage intitulé Stendhal, le « bord de l'eau » et la "note secrète », qui met en rapport l'épisode de la "note secrète » dans Le Rouge et le Noir avec de réelles conspirations d'ultras préparant, sous la Restauration, l'invasion du pays par les armées étrangères en cas de menace révolutionnaire. Aragon a lu attentivement cet ouvrage.

11. Jean Prévost, La Création chez Stendhal, Essai sur le métier d'écrire et la psychologie de l'écrivain, ouvrage posthume composé dans le maquis du Vercors, paru en 1951 au Mercure de France.

12. Voir Aragon, La Lumière de Stendhal, op. cit., p. 128 sq. Vittoria Accoramboni fait partie des textes de Stendhal qui ont été traditionnellement réunis sous le titre de « chroniques italiennes ». 
En revanche les travaux de Georges Blin ${ }^{13}$, qui étaient influencés par la phénoménologie et par l'existentialisme, et constituaient une contribution importante aux études stendhaliennes ainsi qu'à la théorie du roman en général, n'ont guère trouvé d'écho auprès d'eux. Les approches formalistes non plus (il faut dire que d'une manière générale, le formalisme a rencontré peu de succès parmi les stendhaliens, bien que le premier des poéticiens soit aussi un grand stendhalien ${ }^{14}$ ).

La Lumière de Stendhal - en particulier les textes de 1948 - vibre du profond optimisme d'après la victoire de 1945. Stendhal y est moins associé à la valeur de l'énergie qu'à la lucidité politique et à l'espérance, qui ne vont pas sans l'enthousiasme du cour, l'amour de la vie, de la femme et de la patrie. La Lumière de Stendhal fait écho aussi bien au cycle d'Elsa qu'au cycle du réel ${ }^{15}$. «Il faut savoir lire, ce qui n'est pas donné à ceux qui n'ont pas de cœur ${ }^{16} »$, écrit Aragon.

Le patriotisme d'Aragon est celui de la Résistance, mais c'est aussi ce patriotisme thorézien de rassemblement, tel qu'il s'exprima fortement dans l'immédiat après-guerre, qui anima le projet d'un parti communiste français faisant valoir son droit à une part essentielle de l'héritage historique de la nation, voire sa plus complète légitimité à poursuivre l'œuvre de la république sociale. Lors du pèlerinage de 1946 à Medan, Aragon achève ainsi son discours : «Rendre justice à Zola, c'est en tirer la leçon, c'est comprendre l'exemple de Zola, c'est continuer Zola, c'est continuer la France ${ }^{17}$. » Zola et Stendhal servent ici (comme Courbet et Hugo dans d'autres textes) une démarche globale de défense et d'illustration du caractère réaliste et prémarxiste d'une certaine tradition politique et culturelle française, et du caractère français du Parti communiste.

Mais la question nationale dans les années 1940 et 1950 n'est pas seulement une question patrimoniale. C'est aussi, en pleine guerre froide, une question d'actualité. Aragon y insiste, Stendhal était un écrivain de l'actuel, et c'est pour cette raison qu'il demeure d'actualité. Il faut savoir lire avec le cœur, mais il faut aussi « savoir lire au temps de la guerre froide ${ }^{18} »$. On peut considérer qu'ici Stendhal est instrumentalisé, dans un ouvrage critique qui est lui-même de circonstance. Aragon fait notamment allusion au projet de la Communauté européenne de défense (proposé par les États-Unis, amendé par Jean Monnet, rejeté par l'Assemblée nationale en 1954), lorsqu'il traite de l'épisode de la «note secrète » dans Le Rouge et le Noir. Il commence

13. En particulier : Stendhal et les problèmes du roman, Paris, José Corti, 1951.

14. Gérard Genette. Voir ses études stendhaliennes dans Figures II et Figures IV.

15. Il est d'ailleurs explicitement question de la mort de Stendhal dans le poème XIV («Pareils à ceux qui s'aiment ») du recueil de 1954, Les Yeux et la Mémoire.

16. Aragon, op. cit., p. 117. Voir aussi à ce sujet l'article consacré à l'adaptation cinématographique de La Chartreuse en 1948, « Stendhal sous les sun-lights », p. 101.

17. Ibid., p. 257. Cf. aussi p. 147 l'idée qu'il faut « savoir lire Stendhal à la lumière nationale». 18. Ibid., p. 116. 
même son étude par ce morceau souvent jugé accessoire du Rouge, le récit de la conspiration ultra aux commandes de laquelle s'est placé le marquis de La Mole. Les hautes classes prêtes à livrer la patrie aux armées étrangères par peur de la Révolution, Stendhal comprenant et dénonçant cela l'année même de la Révolution (1830), et prouvant par là sa foncière solidarité avec elle et son patriotisme démocratique : voilà qui fait directement écho à l'opposition communiste à la CED. Voilà qui est fait aussi pour rappeler l'atmosphère des années 1930 et les trahisons de 1940 à ceux qui accusent le PCF d'être le parti de l'étranger ${ }^{19}$. À la lecture d'un tel ouvrage, comme à la lecture de L'Humanité, les lecteurs communistes trouvent la confirmation qu'ils peuvent concilier l'horizon bolchevique et l'horizon français au sein d'un parti qui veille étroitement aux intérêts de la nation.

Le geste d'Aragon consiste également à revendiquer un héritage littéraire désormais classique, celui des écrivains engagés du XIX ${ }^{e}$ siècle, du réalisme partisan $^{20}$. Il situe son œuvre de romancier, et avec elle celle du PCF, dans le droit fil de ces «passionnés de la vérité 》 résistant aux mensonges et dénonçant les combines de l'aristocratie et de la bourgeoisie.

Le triptyque Stendhal-Zola-Aragon, qui se forme dans La Lumière de Stendhal, dessine une filiation plus complexe qu'il n'y parait. Il agence l'esprit des Lumières, qui est dans le titre, avec le naturalisme zolien, le classicisme stendhalien avec l'avant-garde du roman réaliste incarnée par Aragon lui-même, le roman réaliste prosaïque et la poésie lyrique qu'Aragon a par ailleurs entrepris de renouveler, comme d'autres poètes communistes $^{21}$.

Une définition de l'écrivain engagé en ressort, qui est contemporaine de celle de Sartre, mais sensiblement différente de celle-ci. Il ne faut pas distinguer « l'écrivain » du « politique », écrit en substance Aragon à propos de Zola républicain et dreyfusard ${ }^{22}$, signifiant que Zola n'est pas simplement un écrivain qui s'est engagé pour la justice, mais qu'il a été réaliste par esprit de justice. Il souligne également le fait que Zola s'est engagé dans une entreprise collective de justice : qu'il est, à ce titre, une voix dans un

19. Ibid., p. 27.

20. Et non pas neutre. Aragon y insiste beaucoup, en argumentant notamment contre une interprétation qu'il juge naïve de la métaphore récurrente chez Stendhal du roman comme simple « miroir » de la réalité, et des gloses à cette métaphore dans les textes de Stendhal lui-même (en substance : ce n'est pas ma faute si le miroir reflète la boue du chemin; mon roman ne pouvait être autrement fait). Pour Aragon, Stendhal est au contraire un écrivain engagé et il ne saurait être question de prendre ces affirmations au premier degré. Il faut y reconnaître une manœuvre rhétorique de l'auteur, visant à le protéger de l'accusation (vraie) de partialité et d'hostilité critique à la société bourgeoise. Stendhal ne doit pas être cru quand il veut passer pour « naturaliste » (dans la terminologie d'Aragon).

21. Pablo Neruda notamment.

22. Voir Aragon, op. cit., p. 247 sq. 
groupe, un partisan. Un livre sur Stendhal seulement n'eût pas permis de bâtir une telle figure.

Cependant, l'isolement de Stendhal a également son importance ici. Car La Lumière de Stendhal exprime aussi une position antidogmatique au sein du monde communiste. En défendant Stendhal, Aragon défend un écrivain bourgeois, qui n'a pas été socialiste, encore moins " prolétarien », et ne pouvait pas l'être. L'auteur assume une position dialectique et hétérodoxe, dans la mesure où il explicite son refus d'une littérature qui ne serait que l'illustration « du bien selon les communistes ${ }^{23}$ ». Ce net rejet de l'orthodoxie stalinienne dans les lettres n'est accompagné d'aucune critique de Staline et du stalinisme. Mais tout en étant le défenseur et le passeur de la littérature soviétique en France (il va bientôt créer et diriger chez Gallimard la collection « Littératures soviétiques »), Aragon prend nettement position contre l'orthodoxie esthétique et contre une certaine forme de populisme marxiste-léniniste. Le pluriel du titre de cette collection est significatif à cet égard.

Si l'on rapporte maintenant sa contribution au contexte proprement stendhalien, la ligne de force du propos d'Aragon est d'affirmer le caractère intrinsèquement politique, de bout en bout politique, des textes stendhaliens. Du coup les œuvres privilégiées sont Le Rouge, Lucien Leuwen, dans une moindre mesure Lamiel, et les pamphlets, notamment D'un nouveau complot. Ce dernier texte, qui est d'une rhétorique un peu alambiquée ${ }^{24}$, se trouve résumé à une seule brève citation, qu'Andrieu reprendra telle quelle, qui affirme l'existence d'une classe pensante face à la classe industrielle; donc (est-il aisé de gloser) d'une classe pensante consciente d'être une classe, dans une société de classes, et d'être engagée dans une lutte de classe ${ }^{25}$. Stendhal interprète lucide de l'histoire sociale de son temps, Stendhal « présociologue », pour reprendre le titre d'un récent séminaire balzacien ${ }^{26}$, voilà l'idée majeure d'Aragon. C'est, appliquée à Stendhal,

23. «Cette conception séparatiste, écrit Aragon, est celle de l'anticommunisme, ce n'est pas celle du parti communiste, pour autant que je sois renseigné à ce sujet » (ibid., p. 147). 24. Pour un bon commentaire, équilibré et contextualisé, de ce texte, voir notamment Geneviève Mouillaud, « De Henri Beyle à Stendhal » et « Stendhal et les problèmes de la société », Europe, no 519-520-521, 1972, respectivement p. 16-26 et p. 64-78.

25. Andrieu cite ce passage dans Stendhal et le bal masqué (op. cit., p. 116) : « La classe pensante, mesurant avec soin son estime sur l'utilité, préfère souvent un guerrier, un habile médecin, un savant avocat qui, sans espoir de salaire, défend l'innocence au plus riche fabricant qui importe des machines et emploie dix mille ouvriers. Pourquoi? C'est que pour arriver à une haute estime il faut en général qu'il y ait sacrifice de l'intérêt à quelque noble but.» Il souligne également p. 115 cette phrase du pamphlet de 1825, qui résume la position ironique de Stendhal à l'égard du libéralisme et sa satire des industriels : « Les marchands d'argent ont besoin d'un certain degré de liberté... mais dès que le 8 pour cent se présente, le banquier oublie bien vite la liberté. »

26. Sous la direction d'Andrea del Lungo et Pierre Glaudes dans le cadre du séminaire dix-neuviémiste Paris 3 / Paris 4. L'angle que choisit Aragon pour développer une analyse 
l'analyse de Balzac par Engels ${ }^{27}$. C'est la pierre marxiste dans le jardin du beylisme égotiste, du beylisme de " l'énergie » et du beylisme hédoniste. Yves Ansel, récent éditeur du Rouge dans la nouvelle édition de la Pléiade, est à cet égard l'héritier direct des lectures communistes de Stendhal.

C'est Le Rouge et le Noir qui est privilégié par Aragon (comme par Y. Ansel). Le vif et sentimental attachement à La Chartreuse de Parme (le titre phare du stendhalisme barrésien, le chef-d'œuvre intouchable et le plus consensuel de Stendhal) est aussi très perceptible chez Aragon, mais il ne donne pas lieu à des analyses précises ni à un enthousiasme théorique.

Stendhal apparait, à la lecture d'Aragon, comme un patrimoine littéraire et intellectuel considérable, et donc comme une position stratégique qu'il importe de conquérir. Et manifestement, ce qui ne peut pas être dit de Balzac, peut l'être de Stendhal : c'est un écrivain de gauche, donc de la gauche. Le cas Stendhal passe, du coup, pour moins ambivalent que celui de Balzac. Sans doute, ce dernier manifeste une vive sympathie pour les démocrates, et surtout il a fait, par sa lecture des stratégies sociales, économiques et politiques des bourgeoisies et des aristocraties de son temps, l'objet de toute l'attention d'Engels. Mais il est de droite, et la gauche le sollicite sans pouvoir le « récupérer ». En revanche Stendhal, dont les « compétences » sont comparables, dont le matérialisme est jugé lucide et performant, a toujours manifesté son hostilité à l'Église, à la monarchie et à l'aristocratie financière. Pour ces raisons il apparaît comme compatible avec le marxisme-léninisme.

On retrouve chez Andrieu les lignes de force du propos d'Aragon. Seulement on n'est plus, en 1983, dans une phase de conquête. On est au bout d'un cycle. L'objet d'Andrieu, moins polémique, est de présenter un auteur du « patrimoine de gauche » à un public de non-spécialistes. Plus qu'Aragon, il présente l'auteur, l'homme; mais les éléments biographiques qu'il choisit vont tous dans le même sens : ils mettent en évidence l'attachement de Stendhal à la République et à Bonaparte, indiquent qu'il a réprouvé

marxiste du Rouge est, curieusement, la comparaison récurrente de Julien avec Tartuffe. Aragon y lit non pas une évaluation péjorative du personnage, mais l'indice d'une analyse profonde de l'éthos du dominé, contraint au masque, dans la société de classes. Il y voit même, de la part de Stendhal, une relecture renversante de Molière : Tartuffe est bien le héros du Tartuffe. Andrieu et Barbéris reprendront cette paradoxale analyse. Antoine Vitez aussi (et ce ne saurait être par hasard), dans sa mise en scène de 1978. On aurait là, de Stendhal à Vitez, en passant par Aragon, une intéressante filiation.

27. Lukacs évoque Stendhal dans des termes voisins dans Balzac und der französische Realismus (Berlin, Aufbau-Verlag, 1952). Il distingue le « grand » réalisme, qui contient une analyse de la structure sociale (celui de Balzac et de Stendhal) et le petit (celui de Flaubert et de Zola), qui privilégie les phénomènes de surface et les détails : Stendhal est un grand réaliste, qui va droit aux principes. Ses fameux « petits faits vrais » ne sont pas simplement le rendu des phénomènes; ils veulent signifier la substance des choses. 
Napoléon ${ }^{28}$, illustrent son dédain de la Restauration, puis son dégoût de l'aristocratie bancaire. Stendhal est présenté comme le fils de la Révolution.

Moins sélectif qu'Aragon, Andrieu ne gomme pas les fameuses phrases où Stendhal explicite ce qui le sépare irrémédiablement du peuple ${ }^{29}$, mais il en propose une interprétation contextualisée qui relativise le mépris perceptible dans ces affirmations. Il insiste surtout sur toutes les compensations et consolations qu'un authentique démocrate peut trouver par ailleurs dans le corpus stendhalien ${ }^{30}$, et qui tendent à démontrer que, dans l'esprit de Stendhal, les happy few ne sont pas nécessairement des gens riches et oisifs, mais peuvent aussi être des travailleurs. C'est là un point capital dans la perspective d'Andrieu, bien plus que dans celle d'Aragon, que la littérature de Stendhal puisse être lue comme une littérature démocratique. Andrieu souligne à cet égard en note le sort très favorable des œuvres de Stendhal en URSS, pays qui « semble battre tous les records [d'édition de Stendhal] ${ }^{31} »$.

Le fait est que Stendhal a eu un lectorat d'ouvriers, en France comme en URSS, ainsi d'ailleurs qu'un lectorat de paysans, sinon par sa propre vertu, du moins grâce à l'école; mais, en vérité, il n'a jamais envisagé explicitement qu'il pût en avoir un. Il est clair qu'Andrieu a tendance à « gauchiser » l'attitude de Stendhal à l'égard du peuple. Le peuple n'intéresse profondément Stendhal que dans ses manifestations politiques les plus hérö̈ques, ou lorsqu'il donne sa vie sur les champs de bataille. Ou bien lorsqu'il fait preuve de bonté et en particulier d'hospitalité, ou de simple gentillesse, par exemple pour l'enfant qu'il a été (Stendhal rejoint Rousseau à cet égard). Ou bien, ce qui est tout autre chose, lorsqu'il est impliqué dans d'intéressantes et surprenantes tragédies privées révélant une grande vitalité passionnelle (les affaires Berthet et Lafargue qui inspirèrent Le Rouge, la criminalité dans le peuple italien, qui participe du tableau romanesque que Stendhal propose de l'Italie dans ses fictions, dans ses récits de voyage et dans ses écrits sur l'art). Autrement, Stendhal ne s'intéresse guère au travail, ni tellement au langage populaire, ni à la culture populaire, pas

28. Ce qui est vrai, mais il faudrait préciser dans quels textes, dans quels types de textes et à quel moment de la vie de Stendhal, qui a tout de même servi l'Empire jusqu'en 1814. Dans ses textes destinés à la publication, Stendhal défend systématiquement la mémoire de l'empereur. Il est, à cet égard, sur les mêmes positions que Béranger (dont il avait la faiblesse de penser qu'il était le grand poète français vivant) et que la gauche «nationale ». 29. Andrieu cite notamment cette fameuse phrase tirée de La Vie de Henry Brulard: « J'aime le peuple, je déteste les oppresseurs, mais ce serait pour moi un supplice de tous les instants que de vivre avec le peuple » (René Andrieu, Stendhal ou le Bal masqué, op. cit., p. 79).

30. Andrieu évoque p. 82 sq. la figure de Ferrante Palla dans La Chartreuse de Parme, Julien Sorel lui-même, le récit par Stendhal des combats de juillet 1830, ou la figure de Lambert dans le Brulard. La littérature stendhalienne comporte de nombreux autres héros d'extraction populaire, qui, étonnamment, n'ont jamais fait l'objet d'une étude spécifique. 31. Soit « de 1918 à 1967, 117 éditions en 17 langues totalisant 10 millions 650000 exemplaires dont 12 millions en langue russe » (ibid., p. 12). 
plus en Italie qu'en France (en tout cas pas au point d'en faire, comme Hugo à la même époque, une matière romanesque). La misère dans les classes populaires n'est pas pour lui une préoccupation centrale, même s'il ne l'ignore pas et la signale clairement dans Lucien Leuwen, même s'il est conscient des conditions d'existence atroces du prolétariat anglais, même s'il évoque brièvement le sort des jeunes ouvrières de M. Rênal au premier

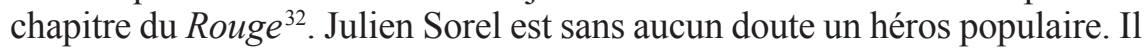
est clairement visé et expliqué comme tel par le narrateur, et perçu comme tel par les autres personnages. Et c'est en partie pour cela qu'il a horrifié la critique réactionnaire du XIX ${ }^{\mathrm{e}}$ siècle. Mais il n'évolue pas dans un milieu populaire, et se distingue autant, et même davantage, du peuple, que des aristocrates. Le peuple comme milieu est au moins trois fois dévalué dans le roman, par le narrateur et par le héros : d'abord à travers la figure du père et des frères de Julien, ensuite au travers des paysans qui accueillent dans un enthousiasme et une piété stupides la visite d'un roi étranger à Verrières, enfin à travers la médiocrité, la saleté et la bassesse congénitales des petits séminaristes de Besançon. C'est pourquoi Julien a été souvent regardé comme une figure aristocratique. Le bruit court, dans le roman, qu'il serait le fils naturel d'un noble.

À la différence notable de Victor Hugo, Stendhal n'a jamais cherché à produire une représentation d'un milieu ouvrier (ni d'ailleurs d'un milieu petit bourgeois); d'où la réticence de Zola. Il paraît clair cependant que l'appréciation par Stendhal des événements de novembre 1831 à Lyon (l'insurrection des canuts et sa répression) a été beaucoup plus marquée « à gauche » que celle de Mérimée (mais, dans leur échange épistolaire de cette époque, nous ne disposons que des lettres de Mérimée conservées par Stendhal). Les Mémoires d'un touriste, texte peu connu et insuffisamment exploité, montrent également que Stendhal n'était pas aveugle à l'industrialisation du pays et à l'avènement d'un prolétariat ouvrier ${ }^{33}$. Mais on ne trouve pas chez lui l'équivalent du travail de documentation des premiers socialistes (Flora Tristan par exemple, l'auteur des Promenades dans Londres $(1840)^{34}$, est autrement plus attentive au sort des classes populaires que celui des Promenades dans Rome).

La principale limite des lectures d'Aragon et d'Andrieu est naturellement leur caractère sélectif : elles ne présentent pas tout le corpus et, au sein des œuvres choisies, sélectionnent ce qui correspond à leurs perspectives d'ensemble. Elles ont tendance à présenter comme parfaitement clair ce qui en vérité l'est rarement, comme « lumineux » des textes qui sont souvent ambigus. Aragon, conscient du problème, explique les précautions

32. Voir Geneviève Mouillaud, op. cit. Voir aussi Yves Ansel, Stendhal littéral : Le Rouge et le Noir, Paris, Kimé, 2001.

33. Voir par exemple la partie consacrée à Saint-Étienne.

34. Martine Reid, spécialiste de Stendhal, en a proposé une édition partielle chez Gallimard en 2008. 
rhétoriques, les parenthèses et les énigmatiques paratextes stendhaliens par la peur de la police ${ }^{35}$, jamais comme la marque d'une indécision conceptuelle ou politique.

Les lectures communistes de Stendhal ne sociologisent pas jusqu'au bout leur objet. Elles ne se demandent pas par exemple pour qui Stendhal écrit : le sujet est réglé par les fameuses phrases où l'écrivain pronostique sans se tromper le succès qu'il remportera vers 1880 . Mais la vérité de ce pronostic (qui a si souvent retenu l'attention de la critique) ne devrait pas le soustraire à l'analyse, et plus précisément à une analyse de type sociologique. D'une manière générale, la critique glose la lucidité sociologique de Stendhal, mais l'envisage rarement lui-même en termes sociologiques (alors qu'il constitue, à cet égard, un cas d'un grand intérêt). Stendhal est manifestement pensé à la lumière de Julien Sorel, et à la lumière des premiers chapitres du Brulard, comme un individu qui s'est extrait par son génie et par sa volonté de son milieu et de sa classe. Pourtant, il n'a jamais évolué que dans sa classe d'origine, à moins qu'il visât plus haut. Le principe d'une « solidarité de classe » liant les intellectuels communistes au sort des ouvriers n'a pas d'équivalent chez Stendhal. Il est vrai qu'il y a dans la conception que Stendhal se fait de la société et des rapports sociaux à la fois une part de déterminisme et une part d'indéterminisme, une morale de la volonté et une confiance dans le mouvement caractéristique du libéralisme du XIX ${ }^{\mathrm{e}}$ siècle et de tous les progressismes. Aragon, et quantité d'intellectuels communistes issus de la bourgeoisie, ont pu s'identifier à ces figures foncièrement labiles que sont Julien Sorel et Henry Brulard, et reconnaître dans le parcours de ces deux personnages (hors de l'ombre et vers la lumière) une métaphore de leur propre parcours et de leur propre progressisme. Mais une conversion idéologique ne constitue pas en soi un reclassement (pas plus qu'un conservatisme idéologique ne préserve d'un déclassement).

La principale limite de la critique communiste de Stendhal est celle du beylisme en général, à savoir qu'elles ont fondamentalement la forme d'un récit dont l'écrivain est le héros. Le héros, c'est-à-dire une figure idéalisée et intouchable. Par exemple, elles n'évaluent pas les prises de position négatives de Stendhal à l'égard de ses confrères romantiques, mais se satisfont d'une approche politique un peu courte : qui dit en substance que si Stendhal n'aime pas, par exemple, Lamartine, c'est parce que celui-ci appartient à la droite catholique, sans voir que ce critère n'a pas tellement orienté les goûts littéraires de Stendhal. Le romantisme est caricaturé par les stendhaliens comme il l'est par Stendhal, les auteurs reprenant à leur compte sans beaucoup de ménagement (c'est le cas d'Andrieu notamment) les jugements de l'écrivain. La critique marxiste propose une interprétation politique de

35. Analyse au demeurant convaincante : Stendhal eut en effet à pâtir de la police autrichienne, de la police toscane, de la police romaine, dont un certain nombre de rapports nous sont connus. 
l'isolement de Stendhal dans le champ littéraire de son temps, en insistant sur sa singularité politique et sur la portée critique impérieuse et profondément dérangeante de son analyse de la société bourgeoise. Mais c'est étendre exagérément l'empire de l'idéologie. Stendhal n'est pas le seul écrivain de son temps à tenir sur la société bourgeoise un discours décapant, et la spécificité de son parcours et de son statut dans le champ littéraire de son temps s'explique par d'autres raisons que des raisons politiques. En s'en tenant à cette approche politique, sans jamais envisager par exemple la dimension poétique, linguistique et stylistique du problème, la critique marxiste ne parvient pas à des conclusions satisfaisantes. Pour reprendre la comparaison avec le cas Balzac, il paraît clair que ce n'est pas l'attachement de Balzac à l'Ancien Régime qui peut expliquer, au moment où celui-ci confirme son effondrement et perd un à un ses alliés, que, à la différence de Stendhal, il ait connu une gloire rapide dans toutes les parties de l'échiquier politique, et de plus en plus à mesure que celui-ci, globalement, se gauchisait.

La lecture de Barbéris se distingue des deux précédentes par le ton : Barbéris est polémique de bout en bout. Mais elle s'en distingue aussi politiquement, pour la raison fondamentale qu'elle abandonne toute logique patrimoniale. L'enjeu culturel «national » est perdu de vue. Ce Stendhal-ci a cessé d'être « un classique »; et, ce qui va de pair, il ne saurait plus susciter de consensus, même partiel. La critique de Barbéris se présente comme strictement motivée par la lutte des classes. Elle est à cet égard beaucoup plus caractéristique de la radicalité des années 1960 et 1970 que du communisme national et parlementaire thorézien, même si par ailleurs, en refusant les apports du formalisme (qui intéressent durant sa période d'activité une certaine gauche, y compris d'ailleurs une gauche communiste ${ }^{36}$ ), Barbéris peut faire figure d' " orthodoxe » marxiste. Son texte est l'expression, si l'on peut dire, d'un « communisme de la dissidence », non pas au sens où il serait solidaire des célèbres dissidents soviétiques, mais au sens où il envisage le militantisme communiste sur le mode de la dissidence. Stendhal apparaît ici comme le héraut (et comme le héros) d'une attitude consistant à ne pas jouer le jeu de la démocratie bourgeoise. C'est ce qui rapproche fondamentalement sa posture critique de celle de Michel Crouzet, ancien responsable de la cellule communiste de l'ENS dans l'immédiat aprèsguerre (soit à une époque de stalinisme triomphant), passé ensuite à l'autre extrême. Leur critique se ressent de cette rivalité dans la radicalité qui oppose et qui conjugue à la fois les différentes composantes de l'extrême gauche dans la France des années 1970, et qui oppose et frotte à celles-ci une certaine extrême droite anarchisante et libertaire.

La contribution de Barbéris tranche également sur les précédentes par le caractère apriorique de son traitement de l'histoire. Celle-ci n'y est plus

36. Il faudrait se reporter pour s'en rendre compte aux numéros de La Nouvelle Critique des années 1970 et 1980. 
figurée comme un mouvement. Le lien avec l'hégélianisme et plus encore avec les Lumières est, sinon perdu, du moins devenu moins nécessaire, consciemment ou inconsciemment. À la différence des deux titres précédents, Sur Stendhal n'est ni un morceau de critique « humaniste », ni un morceau de critique progressiste, au sens où la question de "l'avenir » est devenue incertaine, voire complètement opaque (la communication de Barbéris au colloque du bicentenaire, "Stendhal et l'avenir », est d'un grand intérêt à cet égard). La société française de 1825 est pensée comme déjà fortement analogue à celle de 1983, et celle de 1983 comme encore fondamentalement analogue à celle de 1825. Sans distinction de période, elle est pensée comme une sorte de champ de bataille figé. Ce tableau historique, qui s'autorise de Stendhal, est plus simple que celui que propose l'auteur de La Semaine sainte, et plus approximatif : le prolétariat, avec les intellectuels qui en sont solidaires, produit son effort contre toutes les aristocraties, qui sont assimilables les unes aux autres parce que solidaires contre lui en dépit de leurs désaccords de surface; mais cet effort est vain et l'espace démocratique français tient en vérité de la mascarade ${ }^{37}$.

Ces considérations politiques affectent la conception que l'auteur se fait du romantisme. Le mot a fréquemment dans l'ouvrage de Barbéris un sens banal et péjoratif (naïf, bêtement sentimental), quand il ne signifie pas simplement une posture littéraire " de droite ». Avec une certaine arrogance idéologique et morale (de celle précisément qu'Aragon dénonce avec inquiétude dans son texte de 1954, lorsqu'il évoque le discours d'une jeune stalinienne ${ }^{38}$ ), Barbéris plaque sur Stendhal une conception absolument négative de l'histoire, du lien social et de la politique dans « la société bourgeoise ». Cette approche du romantisme et des révolutions romantiques manquées, qui fait abstraction de février 1848, révèle aussi un rapport ambivalent aux classes populaires. Les journées de juillet 1830 paraissent ici peu signicatives sur le plan historique (car avant et après 1830, explique Barbéris, la France est demeurée fondamentalement la même; elle était déjà aux mains de la bourgeoise, elle l'est devenue officiellement). Le peuple représenté par Delacroix, le peuple qui prend la parole chez Hugo dès avant 1830, a donc été relativement insignifiant dans le processus historique du XIX ${ }^{e}$ siècle. Il paraît difficile d'expliquer février 1848 (et d'une manière générale l'histoire politique européenne du $\mathrm{XIX}^{\mathrm{e}}$ siècle et $\mathrm{du} \mathrm{XX}^{\mathrm{e}}$ siècle,

37. Sur le thème de la mascarade démocratique, on trouverait naturellement aussi une importante littérature de dénonciation d'extrême droite. Maurras notamment voyait en Stendhal un maître en la matière. Cette lecture est aussi celle que fait Michel Crouzet. Voir par exemple sa préface à La Chartreuse de Parme, au Livre de poche (2000).

38. Aragon, op. cit., p. 145 sq. Aragon, plus fin historien que Barbéris et que sa jeune correspondante, note par exemple page 146 que « il faut savoir lire Stendhal, c'est-à-dire en fonction de la société où il s'exprimait, de son temps, d'une époque où, si violente qu'elle fût, écrasant déjà les classes travailleuses, la bourgeoisie n'en était pas moins la bourgeoisie montante, c'est-à-dire qu'en elle s'incarnait le progrès social ». 
jusques et y compris 1917 et la naissance des partis communistes d'ailleurs) à partir de la lecture que fait Barbéris de l'histoire de France. Tout se passe comme si, dans cette singulière historiographie, il y avait des moments sacrés (les moments insurrectionnels réussis) et de longues zones infernales, marquées du sceau d'une négativité absolue (celles où la bourgeoisie règne sans partage et annexe « la vie » elle-même).

Dans le récit de Barbéris, Stendhal est celui qui, sous la tyrannie bourgeoise, entre en dissidence totale. Le fait qu'il ait été fonctionnaire quasiment toute sa vie (comme Barbéris), après avoir été militaire, n'entre pas en ligne de compte. Stendhal est aussi celui qui a tout compris : un maître à penser. C'est ainsi qu'il fut en général admiré par le beylisme de droite comme de gauche, si l'on excepte quelques critiques ou penseurs de plus grande envergure, tels que Blin. Stendhal maître à penser, à sentir, à aimer, Stendhal commencement et fin de toute philosophie, et donc Stendhal en boucle : c'est ce qu'il a été pour Martineau, pour Prévost, pour del Litto, pour Crouzet. Le stendhalisme se caractérise par la constante recherche du paroxysme, soit dans l'émotion, soit dans le concept. Il confine toujours à l'hyperbole; il aime le superlatif. En cela il constitue une assez fidèle, mais peu lucide, réplique de son héros.

On peut s'étonner qu'un marxiste tienne un discours formellement comparable à ceux-là. Tout se passe comme si l'étiquette de " communiste » avait correspondu à une différence culturelle moindre que ce que le positionnement politique laissait entendre.

Par exemple, Stendhal constatait dans La Revue de Paris en 1836 que la comédie était devenue impossible ${ }^{39}$. Le fait que cette affirmation soit fausse n'est généralement pas pris en compte par la critique stendhalienne, qui cherche au contraire à en saisir la logique et à en expliquer le bienfondé. Barbéris va plus loin. Pour Stendhal, écrit-il ${ }^{40}$, le roman aussi est devenu impossible, comme le prouve l'inachèvement et la profonde négativité de Lucien Leuwen. De même, d'ailleurs, « le sérieux moderne exclut la poésie $^{41} \gg$. Cette représentation du champ littéraire des années 1830 n'est évidemment pas satisfaisante.

L'intransigeance de Barbéris à l'égard de ceux qui ont échoué, et sa bienveillance à l'égard de ceux qui les jugent, est elle aussi une traduction amplifiée de Stendhal. Qu'on songe par exemple à Julien jugeant avec un certain dédain l'échec des révolutionnaires espagnols et piémontais - l'échec d'Altamira lui-même (au chapitre II, 9). Qu'on songe à

39. On peut lire ce texte par exemple dans le recueil procuré par Antoine de Baecque, Stendhal, Du rire, Paris, Rivages Poche, 2005.

40. Voir Pierre Barbéris, Sur Stendhal, op. cit., p. 113 sq.

41. Ibid., p. 23. Pour Stendhal, la « poésie » est constitutivement inférieure à la prose, seul vecteur fiable de la pensée vraie. La plupart des stendhaliens se croient obligés de le suivre, et même de le croire, sur ce point. 
Lucien (lui qui assista pourtant aux funérailles du général Lamarque), qui dédaigne la camaraderie des républicains de son régiment (vindicatifs, idéalistes, pitoyables). La démocratie américaine? Imbécile, ennuyeuse et vulgaire, dit la Sanseverina. Pas d'opéra, là-bas, pense-t-elle. Les carbonari romains? Faibles, mal organisés, trop irrationnels, pense le héros d'Une position sociale. Rares sont les positions qui échappent et qui résistent à l'acide stendhalien, à droite bien sûr, mais aussi à gauche. Et il se produit le paradoxe suivant, que le critique communiste valorise le discours de la Sanseverina au sujet de la démocratie américaine (et non les aspirations idéalistes de Fabrice), le discours de Lucien au sujet des républicains (et non les républicains), et le discours de Roizand au sujet des carbonari (et non les carbonari). À cet égard, le critique communiste dit exactement la même chose que toute la critique.

Or, dire comme le suggère Barbéris, et toute la critique stendhalienne avec lui : Stendhal écrit cela au sujet de la démocratie américaine, et parce que c'est la démocratie américaine (donc l'autre, voire l'ennemi), est anachronique. Car, au moment où Stendhal écrit, la démocratie américaine est la seule démocratie réelle, c'est la démocratie. Les cabonari ne s'y trompent pas, qui songent, comme Ferrante Palla, comme Fabrice, à y chercher refuge. Au demeurant, le tableau que la Sanseverina et Stendhal font de la démocratie américaine pointe-t-il dans celle-ci un élément qui lui soit spécifique? N'y aurait-il pas lieu de déplorer dans la France démocratique aussi « le respect qu'il faut avoir pour les artisans de la rue, qui par leur vote décident de tout ${ }^{42} \gg$ (fût-on un candidat communiste) ? Ce n'est pas seulement l'Amérique qui pose problème à la Sanseverina (et à Stendhal), c'est, plus fondamentalement, la démocratie elle-même. De ce point de vue, la lecture de Crouzet, par exemple, est plus conséquente que celle de Barbéris. À coup sûr, le parlementarisme n'est pas la tasse de thé de Stendhal. Et à coup sûr Stendhal est attaché - comme son père - à l'héritage aristocratique de la France, qui est absent d'Amérique : littérature, culture, mœurs, morale.

La prouesse de Stendhal est d'avoir vu, écouté et analysé la société de son temps avec un appareillage conceptuel archaïque (au point qu'il a souvent été vu, à tort, mais non sans raison, comme « un homme du $\mathrm{XVIII}^{\mathrm{e}}$ siècle »). Stendhal est un inventeur largement intuitif de la sociologie et du réalisme (et de la psychologie), et Barbéris voit en lui un «matérialiste scientifique ». C'est un contemporain de Niepce, que l'on compare à Hitchcock. C'est se tromper de six générations.

Stendhal a été une référence culturelle majeure parmi les intellectuels communistes français, en particulier dans les générations qui ont connu l'Occupation et qui ont fait la Résistance. Il a fait l'objet, de la part

42. Stendhal, La Chartreuse de Parme, Paris, Le Livre de poche, 2000, p. 177. 
d'Aragon et Andrieu, d'une interprétation et d'une transmission soignées. Il s'agissait d'un stendhalisme et d'un communisme d'éducation, qui participaient plus largement de la constitution d'un patrimoine, c'est-àdire d'abord d'une mémoire (on affirmait une filiation politique nationale : 1792-1793, 1830-31-32-34, 1848, 1871, 1920, 1936, 1945, dans laquelle le PCF occupait la position terminale et donc la position logique par excellence), mais aussi de la constitution d'un corpus de références littéraires et culturelles classiques, qui éclairait et qui légitimait l'action des intellectuels engagés dans ou aux côtés du PCF. Enfin le stendhalisme communiste participait du projet plus général de dessiner un domaine d'intervention privilégié pour les intellectuels communistes au sein du champ littéraire national, où la mise en pratique du corps doctrinal du communisme français fût féconde. Quand Aragon écrivait sur Stendhal, il le faisait clairement en tant que militant communiste. On peut même dire qu'il le faisait dans une large mesure pour le Parti.

Mais il est clair que Stendhal intéressait aussi Aragon et Andrieu pour son style, pour son hédonisme et son aristocratique singularité. Stendhal apparaît comme un luxe de gauche. À côté de Hugo et Zola. Et à côté de Balzac, en complément.

Il a trouvé un écho remarquable chez Blum, puis chez Prévost, chez les existentialistes, et chez des intellectuels communistes, parce qu'il formule, singulièrement dans Le Rouge et le Noir, une morale virile de la volonté et du combat. Mais cette morale de la liberté concerne tous les aspects de l'existence et constitue un extraordinaire appel d'air, dont les communistes ont pu sentir qu'ils représentaient également la promesse. En rupture nette et courageuse de ce point de vue avec la philosophie de sa classe (comme Aragon lui-même), Stendhal professe un mépris profond du capital, ainsi que le bien-fondé, voire le caractère vital, d'un rapport radicalement non-bourgeois à l'argent. La satire du mariage bourgeois, le féminisme de Stendhal, l'ironie systématique à l'égard des mœurs bourgeoises et de la religion, sont certains de ces traits majeurs de la « philosophie » stendhalienne, qui collent avec la morale privée des communistes.

Ces aspects "privés », et même l'érotisme et la morale sexuelle de Stendhal ont une importance plus grande encore chez Barbéris. Cependant le rapport de Barbéris à Stendhal est beaucoup plus ambivalent, bien qu'il soit moins nuancé, que celui de ses prédécesseurs. Chez Barbéris, Stendhal a changé de statut : d'outil pour une meilleure compréhension du monde, il est devenu le modèle d'une forme de révolte lucide mais sans illusion. Cette analyse est aussi, en partie, celle de Crouzet, qui appartient à peu près à la même génération que Barbéris. À comparer Aragon et Andrieu d'une part, Barbéris et Crouzet d'autre part, on perçoit un net et spectaculaire changement d'époque. On sent que les premiers, contrairement aux seconds, ont été portés par un immense espoir et par l'expérience des guerres et des luttes. On voit que, contrairement aux seconds, ils partagent avec Stendhal 
l'expérience de la révolution et de la guerre qu'il définit dans le Brulard comme l'origine de son expérience et de sa pensée. Ce qui est prégnant chez les seconds, c'est la dénonciation d'une normalité étouffante et de cet ennui qui est peut-être le thème central de Stendhal sous la monarchie de Juillet. Le stendhalisme de Barbéris, plus encore que celui de Crouzet, exprime les affres de l'ennui et d'un profond dégoût. Il faut, pour que de tels textes soient produits par un savant marxiste, qu'une singulière angoisse l'ait saisi, en tant que marxiste et en tant que communiste. On sent que la société française est en passe de devenir, pour un révolutionnaire, une terre de renoncement et de ressentiment. Ni Aragon ni Andrieu, qui sont d'un autre âge, ne pouvaient écrire ce genre de choses, ni sur l'histoire de France, ni sur la littérature, ni sur Stendhal en particulier. Il s'agirait à présent d'évaluer et de comprendre ce qui nous sépare de l'époque où Barbéris et Crouzet ont élaboré leurs thèses. Il s'agirait de comprendre pourquoi elles ont, à nos yeux, à leur tour perdu de leur utilité et de leur pertinence.

François Vanoosthuyse

Université Paris 3 - CRP19 